\title{
Topological $Z_{2}$ invariant in Kitaev spin liquids: Classification of gapped spin liquids beyond projective symmetry group
}

\author{
Masahiko G. Yamada (1* \\ Department of Materials Engineering Science, Osaka University, Toyonaka 560-8531, Japan \\ and Institute for Solid State Physics, University of Tokyo, Kashiwa 277-8581, Japan
}

(Received 8 May 2020; accepted 8 December 2020; published 4 January 2021)

\begin{abstract}
A projective symmetry group (PSG) has been regarded as a classification theory of spin liquids. However, it does not include a symmetry-protected topological order of fermionic spinon excitations, and thus the classification of gapped spin liquids is incomplete. We demonstrate the classification beyond PSG by utilizing the Kitaev model on the square-octagon lattice, where two gapped spin liquids are distinguished by a topological $Z_{2}$ invariant. This $Z_{2}$ invariant can be defined solely by the time-reversal and translation symmetries on condition that the time-reversal symmetry is implemented projectively. Thus, it is a hidden class of topological Kitaev spin liquids with helical edge states, which has been ignored for a long time. This suggests that there exists an unknown classification scheme of gapped spin liquids beyond PSG.
\end{abstract}

DOI: 10.1103/PhysRevResearch.3.L012001

Introduction. A projective symmetry group (PSG) [1] has been regarded as a classification theory of quantum spin liquids [2]. This theory is based on the standard mean-field treatment of spin liquids. In quantum spin liquids, the symmetries of the mean-field Hamiltonian and the original spin model can be different. The mean-field Hamiltonian breaks the original symmetry and the symmetry is restored by an additional gauge transformation. The structure of the gauge transformation is called PSG, which characterizes the spin liquid.

Despite its popularity, PSG does not include a topological insulator/superconductor property [3-6] of a fermionic spinon excitation (FSE). FSE potentially possesses a symmetry-protected topological (SPT) order [7]. The existence of an SPT phase in FSE suggests the emergence of a novel class of symmetry-enriched topological (SET) phases in the original spin model. The PSG theory is incomplete as it cannot classify this class of SET phases where FSE has an SPT order [8,9]. In fact, PSG depends on the mean-field approximation of spin liquids [10], which in principle is impossible to treat the property of FSEs directly [11]. We extend the topological transition between two gapped spin liquids with the same PSG, and exactly show that it is a topological transition between two different SET phases by employing the Kitaev model on the square-octagon lattice.

However, PSG still plays an important role in the classification of these phases. This is because it is also impossible for this classification beyond PSG to be described by topological periodic tables. Conventionally, Kitaev models including the

\footnotetext{
*myamada@mp.es.osaka-u.ac.jp
}

Published by the American Physical Society under the terms of the Creative Commons Attribution 4.0 International license. Further distribution of this work must maintain attribution to the author(s) and the published article's title, journal citation, and DOI. one on the honeycomb lattice [11] with a time-reversal symmetry are regarded as class BDI in the topological periodic table, which is trivial in two dimensions and cannot host an SPT order $[12,13]$. Thus, naively there is no topological $Z_{2}$ invariant in Kitaev spin liquids by this free-fermionic classification. On the other hand, the situation is different in the case of the square-octagon lattice [14-16]. This is because the action of the time-reversal symmetry is nonlocal in the $k$ space $[17,18]$, which potentially makes the conventional topological periodic table classification inapplicable. This exotic PSG may allow another classification of topological $Z_{2}$ invariants inside the same class of PSG owing to the projective nature of symmetries.

It is important to note that the nontriviality of PSG in the square-octagon model does not automatically mean that Majorana fermions host a nontrivial SPT order. There is still an additional topological $Z_{2}$ invariant not included in the PSG classification. Indeed, the Kitaev model on the square-octagon lattice has two gapped phases separated by a gapless line. While these two phases have the same PSG and are indistinguishable in the conventional scheme, we define a $Z_{2}$ invariant which can distinguish between them and demonstrate the topological phase transition between these two phases.

Our use of the Kitaev model is advantageous to the Heisenberg model. In the Kitaev model, the ground state is exactly solvable and the $\pi$-flux state is guaranteed to be stabilized by Lieb's theorem [19]. In the case of the Heisenberg model, the $\pi$-flux state is just one of many mean-field solutions, and the brute-force approach is necessary in the discussion. In addition, the geometry of the square-octagon lattice is also an important ingredient for the existence of a nontrivial $Z_{2}$ invariant.

The definition of the $Z_{2}$ invariant itself is exotic and we call it a "nonlocal Pfaffian invariant." The discussion follows $\mathrm{Fu}$ and Kane [6], but the definition is slightly different from theirs, reflecting the nontrivial PSG. Due to the nonlocal property of the time reversal, the Fourier-transformed form has an 
additional $k$-space translation in the first Brillouin zone. The original Fu-Kane invariant does not work due to this momentum shift, and a new invariant has to be defined nonlocally in the $k$ space.

Indeed, the topological $Z_{2}$ invariant can be defined solely by the time-reversal and translation symmetries in two or three dimensions, and thus it is a hidden class of topological Kitaev spin liquids, which has been ignored in the previous classification of Kitaev spin liquids [20]. The classification is not only beyond PSG but also beyond the topological periodic table, which assumes no momentum shift for the time-reversal symmetry. Indeed, a $Z_{2}$ nontrivial phase can only exist in the case where the time-reversal symmetry accompanies a momentum shift, and thus the strong correlation in the Kitaev model is a necessary condition. The additional $k$-space translation makes it possible to have a $Z_{2}$ invariant beyond the previous class BDI classification.

In this Letter, we define the topological $Z_{2}$ invariant protected by time-reversal symmetry, and discover edge states in the $Z_{2}$ nontrivial phase. Our theory suggests the existence of a huge number of ignored SET phases described by FSEs with SPT order by extending our results to topological crystalline phases [21].

Lattice. The Kitaev model, originally defined for the honeycomb lattice [11], has bond-dependent anisotropic interactions. Usually bonds on the lattice are colored by three different colors, red, green, and blue. Red, green, and blue bonds have $x$-, $y$-, and $z$-directional Ising interactions, respectively, and the ground state becomes a spin liquid for any tricoordinated lattices, as long as each site is connected to three different types of bonds and the ground-state flux sector is symmetric. This condition holds for both the honeycomb and square-octagon lattices. The Hamiltonian is

$$
H=-J_{x} \sum_{\langle j k\rangle \in x} \sigma_{j}^{x} \sigma_{k}^{x}-J_{y} \sum_{\langle j k\rangle \in y} \sigma_{j}^{y} \sigma_{k}^{y}-J_{z} \sum_{\langle j k\rangle \in z} \sigma_{j}^{z} \sigma_{k}^{z},
$$

where $\sigma_{j}$ are Pauli matrices, and $\langle j k\rangle \in x, y$, and $z$ are red, green, and blue bonds, respectively.

This model can be solved by introducing Majorana fermions [11]. We would not follow the detailed description of how to solve this model, but eventually the problem of solving this spin model is reduced to a free-fermion model coupled to a "magnetic" field called flux with a single Majorana mode $c_{j}$. The Hamiltonian can always be recast to this form,

$$
H_{\text {Majorana }}=\frac{i}{4} \sum_{j, k} A_{j k} c_{j} c_{k}
$$

where $A$ is a real skew-symmetric matrix with the following property,

$$
A_{j k}= \begin{cases} \pm 2 J_{\gamma}, & \langle j k\rangle \in \gamma, \\ 0, & \text { otherwise, }\end{cases}
$$

where the sign is determined as follows. For solid bonds in Fig. 4 in Appendix A, + when $j$ is on the even sublattice and $k$ is on the odd sublattice, while - when opposite. For dashed bonds in Fig. 4 in Appendix A, - when $j$ is on the even sublattice and $k$ is on the odd sublattice, while + when opposite. By diagonalizing this Hamiltonian, we can get the ground-state spectrum and all SPT information is included. (a)

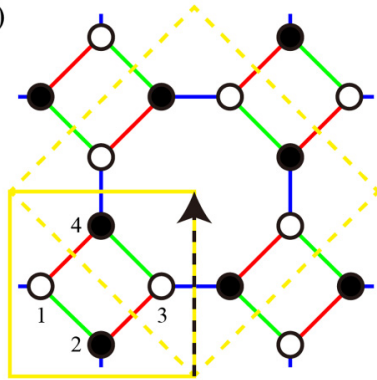

(b)

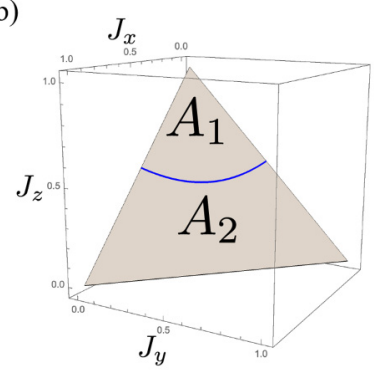

FIG. 1. (a) Kitaev model on the square-octagon lattice. Red, green, and blue bonds have an $x-, y$-, and $z$-directional Ising interaction, respectively, and odd and even sublattices are distinguished by white and black circles, respectively. (b) Phase diagram on the plane $J_{x}+J_{y}+J_{z}=1$ [14]. The $A_{1}$ phase is a topological one with edge states, while the $A_{2}$ phase is not.

Kitaev models on the two-dimensional (2D) squareoctagon lattice [14-16] can actually be defined in many ways, but we use the most symmetric coloring by respecting the translation symmetry of the skeletal structure [see Fig. 1(a)]. In this case, every bond in the same direction has the same color. Lieb's theorem is applicable and the ground-state sector has a $\pi$ flux [19]. Thus, we only consider a $\pi$-flux state in this Letter.

It was found that there are two gapped phases $A_{1}$ and $A_{2}$ in the Kitaev model on the square-octagon lattice [see Fig. 1(b)] [14]. It is important to note that the flux sector and PSG do not change between these two phases, so these two gapped phases are indistinguishable by PSG. These phases are separated by the gapless line with two Dirac cones at $(0,0)$ and $(\pi, \pi)$ in the usual Brillouin zone. The phase boundary can be written as $J_{x}^{2}+J_{y}^{2}=J_{z}^{2}$, so $J_{x}=J_{y}=1 / 4, J_{z}=1 / 2$ is in the $A_{1}$ phase, while $J_{x}=J_{y}=J_{z}=1 / 3$ is in the $A_{2}$ phase [14]. The existence of Dirac cones suggests that the two phases are distinguished by some topological number.

Projective symmetry. Since a $Z_{2}$ gauge is fixed in Eq. (2), the symmetry operation can potentially change the gauge in Majorana fermion systems. Differently from electronic systems, where symmetry actions do not accompany the gauge change, symmetries in Majorana systems often require an additional gauge transformation in order to act within the fixed gauge sector. Such a supplemental gauge transformation sometimes leads to a momentum shift in the $k$ space. Following Refs. [11,17,20], we review the properties of (projective) time-reversal and inversion symmetries in the Kitaev model.

Indeed, the implementation of the most fundamental symmetry operation, time reversal, is exotic with a momentum shift, which changes the topological classification. According to PSG, the time-reversal operator accompanies a gauge transformation which is defined by a sublattice parity $(-1)^{j}$ for the $j$ th site. This fact can be understood as follows. Naively, a candidate time-reversal symmetry is defined by a complex conjugation $K$ as $K c_{j} K=c_{j}$. However, $K$ changes the sign of a hopping term $i c_{j} c_{k}$, and thus needs to be supplemented by some gauge transformation. In bipartite lattices, the simplest choice of the gauge transformation is the sublattice parity $(-1)^{j}$, which is 1 for the even sublattice and -1 for the odd sublattice. Since for each hopping term $j$ and $k$ lie on different 
sublattices, this gauge transformation always supplements the sign caused by the complex conjugation.

Therefore, the time-reversal operation can be written as $\Theta=(-1)^{j} K$. This fact causes an interesting effect on the square-octagon lattice. Since in the square-octagon lattice the sublattice parity is not commensurate with the translation symmetry [see Fig. 1(a)], the unit cell shown by the solid yellow line is effectively enlarged to the dashed yellow line. If we use the original unit cell, an additional $k$-space translation with a wave vector $\boldsymbol{k}_{0}=(\pi, \pi)$ is necessary after the Fourier transformation. Thus, due to the projectiveness the action of the time reversal in the $k$ space becomes between $\boldsymbol{k}$ and $\boldsymbol{k}_{0} \boldsymbol{-} \boldsymbol{k}$ [17]. This exotic nature of time reversal is relevant to the classification of SPT phases. The topological classification becomes different from the usual class BDI, resulting in the possibility of the existence of an exotic phase impossible in the classification based on the topological periodic table.

As for the (bond-centered) inversion symmetry, the gauge transformation does not enlarge the unit cell. The inversion is supplemented by the following gauge transformation $G_{I}$ in the $k$ space. The site index from 1 to 4 for the following matrices is shown in Fig. 1(a),

$$
G_{I}=\left(\begin{array}{cccc}
-1 & 0 & 0 & 0 \\
0 & 1 & 0 & 0 \\
0 & 0 & 1 & 0 \\
0 & 0 & 0 & -1
\end{array}\right),
$$

with a site change

$$
P_{\text {phys }}=\left(\begin{array}{cccc}
0 & 0 & 1 & 0 \\
0 & 0 & 0 & 1 \\
1 & 0 & 0 & 0 \\
0 & 1 & 0 & 0
\end{array}\right) .
$$

Thus, the total operator $P$ for the inversion in the $k$ space is

$$
P=G_{I} P_{\text {phys }}=\left(\begin{array}{cccc}
0 & 0 & -1 & 0 \\
0 & 0 & 0 & 1 \\
1 & 0 & 0 & 0 \\
0 & -1 & 0 & 0
\end{array}\right) .
$$

While the inversion $P$ connects $\boldsymbol{k}$ and $-\boldsymbol{k}$ in the $k$ space as usual, it holds that $P^{2}=-1$.

Symmetry indicator approach. We quickly construct a FuKane symmetry indicator [6] which distinguishes the two phases. The existence of the symmetry indicator automatically proves that the two phases are separated as long as the inversion symmetry is protected, but later we will find that the inversion symmetry is not necessary and actually the same invariant can be defined solely by the exotic time-reversal symmetry.

Assuming the inversion symmetry $P$ with $P^{2}=-1$, the inversion eigenvalues $\pm i$ can be defined at every inversioninvariant momentum (IIM), $\Gamma_{1}=(0,0), \Gamma_{2}=(\pi, 0), \Gamma_{3}=$ $(\pi, \pi)$, and $\Gamma_{4}=(0, \pi)$ (see Fig. 2). We note that these IIM are not time-reversal invariant, and thus here they are not called time-reversal-invariant momenta.

Since $[\Theta, P]=0$ and $\Theta$ is antiunitary, the time reversal flips the sign of the inversion eigenvalue. If we define the inversion eigenvalue of the $\alpha$ th Bloch eigenstate $\left|u_{\alpha, k=\Gamma_{i}}\right\rangle$ as $i \xi_{\alpha}\left(\Gamma_{i}\right)$ with $\xi_{\alpha}\left(\Gamma_{i}\right)= \pm 1(i=1, \ldots, 4), \Gamma_{1}$ and $\Gamma_{3}\left(\Gamma_{2}\right.$ and $\left.\Gamma_{4}\right)$ are always related with each other. In fact, $\xi_{\alpha}\left(\Gamma_{1}\right)=$

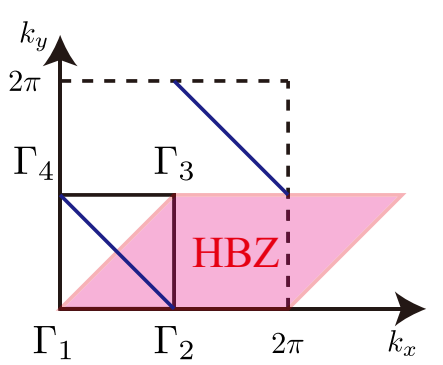

FIG. 2. Inversion invariant momenta $\Gamma_{i}(i=1, \ldots, 4)$ are shown. A half Brillouin zone (HBZ) is represented by the pink shaded region. Blue solid lines represent the subsystem where the DIII topological number is defined.

$-\xi_{\alpha}\left(\Gamma_{3}\right)$ and $\xi_{\alpha}\left(\Gamma_{2}\right)=-\xi_{\alpha}\left(\Gamma_{4}\right)$, so the $Z_{2}$ invariant should be defined only for $\Gamma_{1}$ and $\Gamma_{2}$ to erase the redundancy. A candidate $Z_{2}$ invariant to distinguish two phases is

$$
\delta=\prod_{\alpha=1}^{N} \prod_{i=1}^{2} \xi_{\alpha}\left(\Gamma_{i}\right)
$$

where $N=2$ is the number of occupied bands.

By checking the inversion eigenvalues, the $A_{1}$ phase indeed has $\delta=-1$ and is topologically nontrivial, while the $A_{2}$ phase has $\delta=1$. Thus, from this simple guess, we conclude that the $A_{1}$ phase is topological, i.e., a new SET phase, and the $A_{2}$ phase is not. This $Z_{2}$ invariant $\delta$ is related to a nonlocal Pfaffian invariant, as discussed in Appendix B.

Dimensional reduction approach. In the previous section, we intensively used the inversion symmetry to define the $Z_{2}$ invariant. However, the inversion symmetry is actually unnecessary, and we seek a definition which will not require the inversion eigenvalues in this section.

Another way of evaluating this topological number is to think that the time-reversal symmetry effectively breaks the translation symmetry, and the unit cell is enlarged by this "weak symmetry breaking" [11]. In this enlarged unit cell, a more explicit construction of the $Z_{2}$ invariant is possible.

This picture is indeed similar to the one used in the type IV magnetic space group [22], which is useful to connect the $Z_{2}$ invariant above to the previously discovered phases of topological insulators/superconductors. It is closely related to antiferromagnetic topological insulators $[23,24]$ and we can follow their theory to construct a similar invariant in the enlarged unit cell.

In the enlarged unit cell, the time-reversal symmetry is no longer projective, but rather we need an additional "half translation" to restore the original crystalline symmetry. Since the time reversal in the Kitaev model accompanies the gauge transformation which flips signs for one of the sublattices, this sign flip is restored only by the half translation which switches the sublattice parity. There are many half-translation vectors, but we choose $\boldsymbol{D}=(1 / 2,1 / 2)$, as shown in the black dashed arrow in Fig. 1(a), for simplicity. These two operations have the same gauge transformation, so the combination of the two, denoted by $\Theta_{S}$, can be written without a gauge transformation as $\Theta_{S}=T_{D} K$, where $T_{D}$ is a half translation without a gauge transformation. 
In the $k$ space, $\Theta_{S}$ is a new symmetry defined for the whole Brillouin zone. Especially, $\Theta_{S}^{2}=e^{2 \boldsymbol{D} \cdot \boldsymbol{k} i}$, and $\Theta_{S}^{2}=-1$ when $2 \boldsymbol{D} \cdot \boldsymbol{k}=\pi$. Thus, we have a Kramers degeneracy on the line $2 \boldsymbol{D} \cdot \boldsymbol{k}=\pi$, passing $(0, \pi)$ and $(\pi, 0)$. On this onedimensional (1D) subsystem, shown as blue solid lines in Fig. 2, $\Theta_{S}$ serves as an effective time-reversal symmetry of the $1 \mathrm{D}$ class DIII [24]. Following the definition of the $Z_{2}$ invariant for the 1D topological superconductor in class DIII [25], the $Z_{2}$ invariant is again defined by a formula Eq. (8) within the approach using an enlarged unit cell.

First, we define $\{|\alpha\rangle\}_{\alpha}$ as occupied Bloch functions at $(0, \pi)$, and $\{|\tilde{\alpha}\rangle\}_{\alpha}$ as occupied Bloch functions at $(\pi, 0)$. We note that the basis choice for occupied states is arbitrary at each $k$ point. Then, we define matrices $\left[w_{S}(0, \pi)\right]_{\alpha \beta}=$ $\left\langle\alpha\left|\Theta_{S}\right| \beta\right\rangle$ and $\left[w_{S}(\pi, 0)\right]_{\alpha \beta}=\left\langle\tilde{\alpha}\left|\Theta_{S}\right| \tilde{\beta}\right\rangle$. Intermediate states are also necessary to restore the basis independence as follows,

$$
\begin{gathered}
\delta=\left(\operatorname{det} U^{K}\right) \frac{\operatorname{Pf}\left[w_{S}(0, \pi)\right]}{\operatorname{Pf}\left[w_{S}(\pi, 0)\right]}, \\
U_{\alpha \beta}^{K}=\left\langle\tilde{\alpha}\left|\left(\lim _{n \rightarrow \infty} \prod_{j=0}^{n} P_{F}\left(\boldsymbol{k}_{j}\right)\right)\right| \beta\right\rangle,
\end{gathered}
$$

where $\boldsymbol{k}_{j}=(j \pi / n, \pi-j \pi / n)$ and $P_{F}(\boldsymbol{k})$ is a spectral projector onto the occupied states at $\boldsymbol{k}$ [25].

If we compute the quantity above, indeed we reproduce $\delta=-1$ for the $A_{1}$ phase and $\delta=1$ for the $A_{2}$ phase. In the calculation we used $n=1000$ points between $(0, \pi)$ and $(\pi, 0)$. This formula is useful not only because it is gauge invariant but also because it only requires the information of the time-reversal symmetry. This proves that the inversion symmetry is not necessary for the phase protection. As we have defined the same invariant in two different ways, we will move on to its topological consequence, edge states [26].

Edge states. It is a well-known fact in the case of antiferromagnetic topological insulators that edge states exist for the nontrivial phase when the surface is terminated in the $\Theta_{S}$-symmetric way [24,27]. This condition is met for almost every boundary termination for the square-octagon lattice. Therefore, we check the existence of edge states with the simplest open boundary condition.

We used a strip geometry by simply repeating the original unit cell of the square-octagon lattice for 25 times along the $y$ direction. The band structure based on this termination is shown in Fig. 3. The helical gapless points correspond to edge states and protected by the time-reversal and particlehole symmetries. We note that each gapless point is pinned at $k_{x}=0$ or $k_{x}=\pi$ because of the time-reversal symmetry.

The meaning of the robust helical edge state cannot be captured within the previous framework of SET phases because both $A_{1}$ and $A_{2}$ phases are $Z_{2}$ topologically ordered states described by the toric code. What makes a difference is the topological band structure of FSEs, and its protection by the time-reversal symmetry. The general classification of such phases is an interesting future problem.

Discussions. Here, we defined a topological $Z_{2}$ invariant for Kitaev models in a certain class, where the time-reversal symmetry accompanies a momentum shift. We gave a definition of the same invariant threefold (including the one

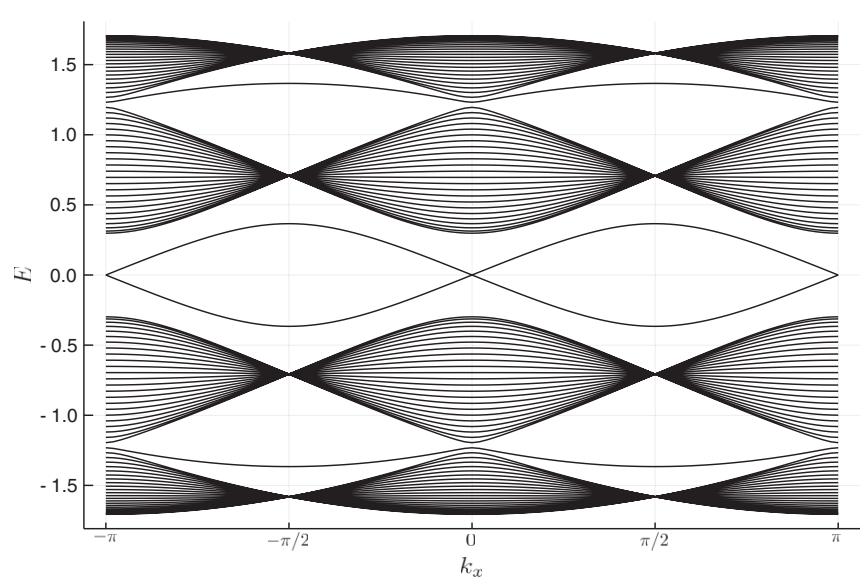

FIG. 3. Band structure for a 100-site strip of the square-octagon lattice. Energy $E$ is shown in the unit of $A_{j k} . J_{x}=J_{y}=1 / 4$ and $J_{z}=$ $1 / 2$, deep inside the $A_{1}$ phase, were used for the calculation.

in Appendix B), and discovered that the nontrivial phase is protected solely by the time-reversal symmetry. We only discussed the 2D system, but the generalization to three dimensions is straightforward because the classification of topological superconductors in class DIII of the 2D subsystem is again $Z_{2}$. The necessary ingredients are the same in both $2 \mathrm{D}$ and 3D, and we need a momentum shift in the $k$ space for the time-reversal symmetry. A $\pi$ flux may also be useful to have an unusual phase. We note the disorder effect in such gapped phases is also an interesting problem [28,29].

We can easily imagine another "beyond PSG" phase, a topological crystalline spin liquid, protected by the crystalline symmetry $[7,30]$. The $(8,3)-n$ lattice is known to have a similar transition between two gapped phases, but the time-reversal symmetry does not accompany a momentum shift [20]. Thus, the Kitaev model on this lattice is a potential candidate for a topological crystalline spin liquid [31,32]. Discovering topological (crystalline) insulators in tricoordinated lattices realizable in iridates or Ru compounds [33-35] is also a future problem.

The conclusion implies that PSG does not give a complete classification of gapped spin liquids. We would need a postPSG theory which can correctly distinguish the SPT orders of FSEs in order to classify gapped spin liquids correctly in the future.

Acknowledgments. We thank V. Dwivedi, D. Else, L. Fu, S. Fujimoto, M. Hermanns, C. Hickey, H. Katsura, T. Morimoto, S. Ono, H. C. Po, K. Shiozaki, and Y. Tada. A part of this work has been done during the visit to Massachusetts Institute of Technology, supported by JSPS. M.G.Y. is supported by the Materials Education program for the future leaders in Research, Industry, and Technology (MERIT), and by JSPS. This work was supported by JST CREST Grant No. JPMJCR19T5, Japan, and by JSPS KAKENHI Grant No. JP17J05736.

\section{APPENDIX A: PROJECTIVE SYMMETRY GROUP}

We present the projective symmetry group (PSG) of the Kitaev model on the square-octagon lattice, assuming the $\pi$ flux mean-field theory [36-38]. We do not include other flux 


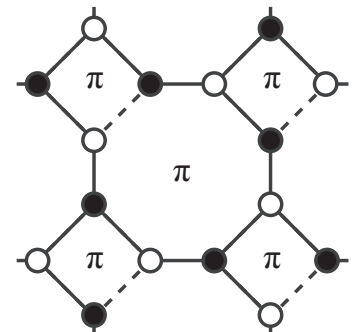

FIG. 4. Square-octagon lattice with a $\pi$ flux. Sublattice $A$ is shown in white circles and sublattice $B$ is shown in black circles. Solid bonds have +1 hopping, while dashed bonds have -1 hopping to make the flux value $\pi$.

sectors because they are not discussed in the main text. We also do not follow Wen's approach to the Heisenberg model [1]. We would not exhaust all possible PSGs in the Kitaev model because in the Kitaev model the exact solution is the only one determined by Lieb's theorem [19]. All the other mean-field solutions are guaranteed to have a higher energy and are of less importance.

First, let us introduce four Majorana operators $c_{j}^{\gamma}$ for the $j$ th site with $\gamma=0, x, y, z . c_{j}^{0}$ corresponds to an itinerant Majorana fermion $c_{j}$ in the main text, so we sometimes omit the superscript 0 for simplicity. After introducing Kitaev's representation $\sigma^{\gamma}=i c^{\gamma} c^{0}$, the Kitaev Hamiltonian is recast into a four-body Majorana Hamiltonian. Its mean-field solution has a form

$$
H_{\mathrm{MF}}=-\frac{1}{8} \sum_{\langle j k\rangle \in \gamma} J_{\gamma}\left(i c_{j} \bar{U}_{j k}^{\gamma \gamma} c_{k}+i c_{j}^{\gamma} \bar{U}_{j k}^{00} c_{k}^{\gamma}\right)+\text { const, }
$$

where $\bar{U}_{j k}^{\mu \nu}=\left\langle i c_{j}^{\mu} c_{k}^{v}\right\rangle$.

At the center of the phase diagram $J_{x}=J_{y}=J_{z}>0$, the solution with a $\pi$ flux is

$$
\bar{U}_{j k}^{\mu \nu}= \begin{cases}\text { const } & \text { for } \mu=v=0 \\ \pm 1 & \text { for } \mu=v=\gamma \text { and }\langle j k\rangle \in \gamma \\ 0 & \text { otherwise }\end{cases}
$$

where the sign of \pm 1 is decided as $+1(-1)$ for a solid (dashed) bond in Fig. $4, j$ always belongs to sublattice $A$ and $k$ belongs to sublattice $B$, and $\bar{U}_{j k}^{\mu \nu}=-\bar{U}_{k j}^{v \mu}$ [38]. In the generic case, $\left|\bar{U}_{j k}^{\mu v}\right|$ will depend on each "bond color," but this does not matter as long as the translation and time-reversal symmetries are not broken and $\bar{U}_{j k}$ stays diagonal.

Assuming all of the relevant symmetries in the main text, i.e., the translation and time-reversal symmetries, we now have three symmetry group (SG) generators: $\mathrm{SG}=$ $\left\langle\left\{\hat{T}_{1}, \hat{T}_{2}, \hat{\Theta}\right\}\right\rangle$. We note that $\hat{T}_{1}$ and $\hat{T}_{2}$ are translations along the $x$ and $y$ directions, respectively, and $\hat{\Theta}$ is time reversal. Here, the meaning of the symmetry operation is slightly different from that in the main text, so we put a hat on to distinguish this mean-field theory from the exact solution.

The gauge chosen in Fig. 4 respects the translation symmetries, which simplifies discussions drastically. The translation along the $x$ direction only changes the sublattice parity, so

$$
\hat{T}_{1}\left(i \bar{U}_{j k}\right)=i \bar{U}_{k j}=-i \bar{U}_{j k} .
$$

The same is true for the $y$ direction,

$$
\hat{T}_{2}\left(i \bar{U}_{j k}\right)=i \bar{U}_{k j}=-i \bar{U}_{j k} .
$$

Finally, since the time-reversal symmetry is antiunitary,

$$
\hat{\Theta}\left(i \bar{U}_{j k}\right)=-i \bar{U}_{j k} .
$$

As described in the main text, the gauge transformation accompanied by these operations is always $G(j)=(-1)^{j}$, where $(-1)^{j}$ is a sublattice parity. If we write this sitedependent gauge transformation as $\hat{G}$, then the PSG for the Kitaev model on the $\pi$-flux square-octagon lattice with a symmetry group $\mathrm{SG}$ is given by

$$
\mathrm{PSG}=\left\langle\left\{\hat{G}_{0}, \hat{G} \hat{T}_{1}, \hat{G} \hat{T}_{2}, \hat{G} \hat{\Theta}\right\}\right\rangle,
$$

where $G_{0}(j)=-1$ generates the invariant gauge group.

An important point is that the discussion here applies to any value of $J_{x}>0, J_{y}>0$, and $J_{z}>0$. Indeed, the $\pi$-flux ansatz is always guaranteed to be the ground state by Lieb's theorem [19], and thus PSG is kept constant in the whole phase diagram of $J_{x}>0, J_{y}>0$, and $J_{z}>0$. In the language of PSG, there is no distinction between phases $A_{1}$ and $A_{2}$ in the main text. They are different only in the sense of the symmetry-protected topological order of fermionic spinon excitations, and therefore we claim it to be a classification of gapped quantum spin liquids beyond PSG.

\section{APPENDIX B: NONLOCAL PFAFFIAN INVARIANT APPROACH}

As mentioned in the main text, we would introduce the third derivation of the $Z_{2}$ invariant, a nonlocal Pfaffian invariant approach. This section is important in the sense that the Pfaffian invariant is modified reflecting the nonlocality of the time-reversal action in the $k$ space. We believe this phenomenon is intrinsic to quantum spin liquids with a nontrivial PSG and thus is of great importance as a guiding principle to explore another topological invariant of quantum spin liquids in the future.

We already found a Fu-Kane-type formula for the classification of a class of Kitaev models in the main text, and thus we can expect that it is related to some Pfaffian invariant [6]. Differently from the topological insulator, a new Pfaffian invariant requires a quantity defined nonlocally in the $k$ space. Thus, we call it a nonlocal Pfaffian invariant, defined from a vector bundle on the reduced (half) Brillouin zone. In this way we can directly connect a Fu-Kane invariant to a Berry phase in this half Brillouin zone (HBZ) (see Fig. 2),

$$
\mathcal{A}(\boldsymbol{k})=-i \sum_{\alpha}\left\langle u_{\alpha, \boldsymbol{k}}\left|\nabla_{\boldsymbol{k}}\right| u_{\alpha, \boldsymbol{k}}\right\rangle .
$$

Although the time reversal itself divides the Brillouin zone into an orbifold by identifying $\boldsymbol{k}$ and $\boldsymbol{k}_{0}-\boldsymbol{k}$, the Brillouin zone is now divided into a $2 \mathrm{D}$ torus by combining the time reversal and the inversion, which identifies $\boldsymbol{k}$ and $\boldsymbol{k}+\boldsymbol{k}_{0}$. Thus, on this "half torus," shown in the pink shaded region in Fig. 2, we can define a smooth twofold degenerate vector bundle of eigenstates, i.e., the Hilbert spaces for Bloch functions $\mathcal{H}_{k}$ and $\mathcal{H}_{\boldsymbol{k}+\boldsymbol{k}_{0}}$ are combined into $\mathcal{H}_{\boldsymbol{k}} \oplus \mathcal{H}_{\boldsymbol{k}+\boldsymbol{k}_{0}}$. Now we can identify two points $\left(k_{x}, k_{y}\right)$ and $\left(k_{x}+\pi, k_{y}+\pi\right)$ in the original Brillouin zone to make an HBZ, and we distinguish 
two positions $\left(k_{x}, k_{y}\right)$ and $\left(k_{x}+\pi, k_{y}+\pi\right)$ by an internal degree of freedom $\tau=\uparrow, \downarrow$, respectively. From now on $\tau$ is regarded as an internal pseudospin, but off-diagonal components about $\tau$ are actually nonlocal in the original $k$ space. On this manifold of HBZ, the time reversal switches the internal degree of freedom, so the time reversal in $\mathrm{HBZ}$ can be written $\Theta_{+}=\tau^{x} \otimes \Theta$.

About the Bloch Hamiltonian $H_{\mathrm{HBZ}}=H(\boldsymbol{k}) \oplus H\left(\boldsymbol{k}+\boldsymbol{k}_{0}\right)$, $H_{\mathrm{HBZ}}$ indeed commutes with $\Theta_{+}$at inversion-invariant momentum (IIM). However, there is another symmetry $\tau^{z}$, which commutes with the Bloch Hamiltonian in the whole HBZ. Thus, we can define another time-reversal symmetry $\Theta_{-}$by $\Theta_{-}=\tau^{z} \Theta_{+}=i \tau^{y} \otimes \Theta$. Here, $\Theta_{-}^{2}=-1$.

The parity time-reversal $\left(\Theta_{+}\right)$operation $\Theta_{+} P$ also acts antiunitarily with a condition $\left(\Theta_{+} P\right)^{2}=-1$, and thus we can use these operators to define a $Z_{2}$ invariant. We define $v_{m n}(\boldsymbol{k})=\left\langle u_{m, \boldsymbol{k}}\left|\Theta_{+} P\right| u_{n, \boldsymbol{k}}\right\rangle$. Here, we newly include $\tau$ indices in $m$ and $n$. Since $\left[\Theta_{+} P, H_{\mathrm{HBZ}}\right]=0$, a matrix $v(\boldsymbol{k})$ is unitary, and from $\left(\Theta_{+} P\right)^{2}=-1, v(\boldsymbol{k})$ is antisymmetric. Thus, the Pfaffian of $v(\boldsymbol{k})$ exists and has a unit magnitude. Its gradient should be related to a Berry phase,

$$
\begin{aligned}
\mathcal{A}(\boldsymbol{k})+\mathcal{A}\left(\boldsymbol{k}+\boldsymbol{k}_{0}\right) & =-\frac{i}{2} \operatorname{Tr}\left[v(\boldsymbol{k})^{\dagger} \boldsymbol{\nabla}_{\boldsymbol{k}} v(\boldsymbol{k})\right] \\
& =-i \boldsymbol{\nabla}_{\boldsymbol{k}} \log \operatorname{Pf}[v(\boldsymbol{k})] .
\end{aligned}
$$

We can easily prove that $\nabla \times\left[\mathcal{A}(\boldsymbol{k})+\mathcal{A}\left(\boldsymbol{k}+\boldsymbol{k}_{0}\right)\right]=\mathbf{0}$, so we choose a gauge so that $\mathcal{A}(\boldsymbol{k})+\mathcal{A}\left(\boldsymbol{k}+\boldsymbol{k}_{0}\right)=\mathbf{0}$. Thus, after the gauge transformation of a form $\operatorname{Pf}[v(\boldsymbol{k})] \rightarrow$ $\operatorname{Pf}[v(\boldsymbol{k})] e^{-i \theta_{k}}$, we can fix a gauge to make $\operatorname{Pf}[v(\boldsymbol{k})]=1$. At the same time, the nontriviality coming from the definition of $\sqrt{\operatorname{det}}$ disappears. The rest is to relate $v_{m n}(\boldsymbol{k})$ to $w_{m n}(\boldsymbol{k})=$

$$
\begin{aligned}
& \left\langle u_{m,-\boldsymbol{k}}\left|\Theta_{-}\right| u_{n, \boldsymbol{k}}\right\rangle, \\
& \quad w_{m n}\left(\Gamma_{i}\right)=-\left\langle\psi_{m, \Gamma_{i}}\left|\left(\Theta_{+} P\right)\left(-\tau^{z}\right) P\right| \psi_{n, \Gamma_{i}}\right\rangle .
\end{aligned}
$$

This is because $P^{2}=-1$. Using an antilinearity of $\Theta$,

$$
w_{m n}\left(\Gamma_{i}\right)=-i \tau_{n}\left(\Gamma_{i}\right) \xi_{n}\left(\Gamma_{i}\right) v_{m n}\left(\Gamma_{i}\right),
$$

where $n$ includes an index $\tau$ and $\tau_{n}\left(\Gamma_{i}\right)$ is its $\tau^{z}$ eigenvalue. First, we note that

$$
\operatorname{Pf}[w]^{2}=\operatorname{det}[w]=\operatorname{det}[v] \prod_{n=1}^{2 N}\left[-i \tau_{n}\left(\Gamma_{i}\right) \xi_{n}\left(\Gamma_{i}\right)\right] .
$$

As we already saw $\xi_{\alpha}\left(\Gamma_{i}\right)=-\xi_{\alpha}\left(\Gamma_{i}+\boldsymbol{k}_{0}\right)$, each pair of states with an opposite $\tau$ has an opposite sign. Then,

$$
\operatorname{Pf}[w]=\operatorname{Pf}[v] \prod_{\alpha=1}^{N}\left[-i \xi_{\alpha}\left(\Gamma_{i}\right)\right] .
$$

Thus, using $\operatorname{Pf}[v]=1$, a Fu-Kane invariant inside HBZ can be computed from the product for two $k$ points $\Gamma_{1}$ and $\Gamma_{2}$,

$$
\delta=\prod_{i=1}^{2} \operatorname{Pf}\left[w\left(\Gamma_{i}\right)\right]=\prod_{\alpha=1}^{N} \prod_{i=1}^{2}\left[-i \xi_{\alpha}\left(\Gamma_{i}\right)\right],
$$

which coincides with the previous definition for $N=2$.

In the final form, it is not apparent that this invariant is still valid when the inversion symmetry is broken. However, if we go back to the definition, the $\operatorname{Pf}[w]$ is multiplied for every IIM inside HBZ, and thus the $Z_{2}$ invariant here can be defined solely by the time-reversal symmetry. We note that $\operatorname{Pf}[w]$ has a meaning only after identifying $\left(k_{x}, k_{y}\right)$ and $\left(k_{x}+\pi, k_{y}+\pi\right)$.
[1] X.-G. Wen, Quantum orders and symmetric spin liquids, Phys. Rev. B 65, 165113 (2002).

[2] X.-G. Wen, Quantum order: A quantum entanglement of many particles, Phys. Lett. A 300, 175 (2002).

[3] C. L. Kane and E. J. Mele, $Z_{2}$ Topological Order and the Quantum Spin Hall Effect, Phys. Rev. Lett. 95, 146802 (2005).

[4] C. L. Kane and E. J. Mele, Quantum Spin Hall Effect in Graphene, Phys. Rev. Lett. 95, 226801 (2005).

[5] L. Fu, C. L. Kane, and E. J. Mele, Topological Insulators in Three Dimensions, Phys. Rev. Lett. 98, 106803 (2007).

[6] L. Fu and C. L. Kane, Topological insulators with inversion symmetry, Phys. Rev. B 76, 045302 (2007).

[7] M. G. Yamada, V. Dwivedi, and M. Hermanns, Crystalline Kitaev spin liquids, Phys. Rev. B 96, 155107 (2017).

[8] Y. Qi, M. Cheng, and C. Fang, Symmetry fractionalization of visons in $\mathbb{Z}_{2}$ spin liquids, arXiv:1509.02927.

[9] Y.-M. Lu, G. Y. Cho, and A. Vishwanath, Unification of bosonic and fermionic theories of spin liquids on the kagome lattice, Phys. Rev. B 96, 205150 (2017).

[10] A. M. Essin and M. Hermele, Classifying fractionalization: Symmetry classification of gapped $\mathbb{Z}_{2}$ spin liquids in two dimensions, Phys. Rev. B 87, 104406 (2013).

[11] A. Kitaev, Anyons in an exactly solved model and beyond, Ann. Phys. 321, 2 (2006).
[12] A. Kitaev, Periodic table for topological insulators and superconductors, in Advances in Theoretical Physics: Landau Memorial Conference, edited by V. Lebedev and M. Feigel'man, AIP Conf. Proc. Vol. 1134 (AIP, Melville, NY, 2009), p. 22.

[13] S. Ryu, A. P. Schnyder, A. Furusaki, and A. W. W. Ludwig, Topological insulators and superconductors: Tenfold way and dimensional hierarchy, New J. Phys. 12, 065010 (2010).

[14] S. Yang, D. L. Zhou, and C. P. Sun, Mosaic spin models with topological order, Phys. Rev. B 76, 180404(R) (2007).

[15] G. Baskaran, G. Santhosh, and R. Shankar, Exact quantum spin liquids with Fermi surfaces in spin-half models, arXiv:0908.1614.

[16] G. Kells, J. Kailasvuori, J. K. Slingerland, and J. Vala, Kaleidoscope of topological phases with multiple Majorana species, New J. Phys. 13, 095014 (2011).

[17] M. Hermanns and S. Trebst, Quantum spin liquid with a Majorana Fermi surface on the three-dimensional hyperoctagon lattice, Phys. Rev. B 89, 235102 (2014).

[18] M. Hermanns, S. Trebst, and A. Rosch, Spin-Peierls Instability of Three-Dimensional Spin Liquids with Majorana Fermi Surfaces, Phys. Rev. Lett. 115, 177205 (2015).

[19] E. H. Lieb, Flux Phase of the Half-Filled Band, Phys. Rev. Lett. 73, 2158 (1994). 
[20] K. O'Brien, M. Hermanns, and S. Trebst, Classification of gapless $\mathbb{Z}_{2}$ spin liquids in three-dimensional Kitaev models, Phys. Rev. B 93, 085101 (2016).

[21] L. Fu, Topological Crystalline Insulators, Phys. Rev. Lett. 106, 106802 (2011).

[22] H. Watanabe, H. C. Po, and A. Vishwanath, Structure and topology of band structures in the 1651 magnetic space groups, Sci. Adv. 4, eaat8685 (2018).

[23] R. S. K. Mong, A. M. Essin, and J. E. Moore, Antiferromagnetic topological insulators, Phys. Rev. B 81, 245209 (2010).

[24] C. Fang, M. J. Gilbert, and B. A. Bernevig, Topological insulators with commensurate antiferromagnetism, Phys. Rev. B 88, 085406 (2013).

[25] J. C. Budich and E. Ardonne, Topological invariant for generic one-dimensional time-reversal-symmetric superconductors in class DIII, Phys. Rev. B 88, 134523 (2013).

[26] Y.-C. Wang, C. Fang, M. Cheng, Y. Qi, and Z. Y. Meng, Topological spin liquid with symmetry-protected edge states, arXiv:1701.01552.

[27] K. Shiozaki, M. Sato, and K. Gomi, Topology of nonsymmorphic crystalline insulators and superconductors, Phys. Rev. B 93, 195413 (2016).

[28] T. Morimoto, A. Furusaki, and C. Mudry, Anderson localization and the topology of classifying spaces, Phys. Rev. B 91, 235111 (2015).

[29] M. G. Yamada, Anderson-Kitaev spin liquid, npj Quantum Mater. 5, 82 (2020).
[30] M. G. Yamada, M. Oshikawa, and G. Jackeli, Emergent SU(4) Symmetry in $\alpha-\mathrm{ZrCl}_{3}$ and Crystalline Spin-Orbital Liquids, Phys. Rev. Lett. 121, 097201 (2018).

[31] V. Dwivedi, C. Hickey, T. Eschmann, and S. Trebst, Majorana corner modes in a second-order Kitaev spin liquid, Phys. Rev. B 98, 054432 (2018).

[32] Y. X. Zhao, Y. Lu, and S. A. Yang, Topological second-order spin-3/2 liquids with hinge Fermi arcs, arXiv:2005.14500.

[33] G. Jackeli and G. Khaliullin, Mott Insulators in the Strong SpinOrbit Coupling Limit: From Heisenberg to a Quantum Compass and Kitaev Models, Phys. Rev. Lett. 102, 017205 (2009).

[34] K. W. Plumb, J. P. Clancy, L. J. Sandilands, V. V. Shankar, Y. F. $\mathrm{Hu}, \mathrm{K}$. S. Burch, H.-Y. Kee, and Y.-J. Kim, $\alpha-\mathrm{RuCl}_{3}$ : A spinorbit assisted Mott insulator on a honeycomb lattice, Phys. Rev. B 90, 041112(R) (2014).

[35] M. G. Yamada, H. Fujita, and M. Oshikawa, Designing Kitaev Spin Liquids in Metal-Organic Frameworks, Phys. Rev. Lett. 119, 057202 (2017).

[36] Y.-Z. You, I. Kimchi, and A. Vishwanath, Doping a spin-orbit Mott insulator: Topological superconductivity from the KitaevHeisenberg model and possible application to $\left(\mathrm{Na}_{2} / \mathrm{Li}_{2}\right) \mathrm{IrO}_{3}$, Phys. Rev. B 86, 085145 (2012).

[37] U. F. P. Seifert, T. Meng, and M. Vojta, Fractionalized Fermi liquids and exotic superconductivity in the Kitaev-Kondo lattice, Phys. Rev. B 97, 085118 (2018).

[38] K. M. O’Brien, Three-dimensional Kitaev spin liquids, Ph.D. thesis, University of Cologne, 2019. 